\begin{tabular}{|c|c|}
\hline \multirow{3}{*}{ 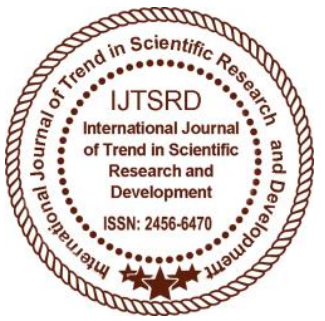 } & $\begin{array}{l}\text { International Journal of Trend in Scientific } \\
\text { Research and Development (IJTSRD) }\end{array}$ \\
\hline & International Open Access Journal \\
\hline & ISSN No: 2456 - 6470 | www.ijtsrd.com | Volume - 1 | Issue - 5 \\
\hline
\end{tabular}

\title{
Comparison between Effectiveness of Hand Arm Bimanual Intensive Training and Repetitive Facilitation Exercises on Upper Limb Functions In Post Stroke Hemiparetic Patients
}

\author{
Dr. Shilpy Jetly \\ Associate Professor, DAV Institute \\ of Physiotherapy and \\ Rehabilitation, Jalandhar, Punjab
}

\author{
Sukhwinder Kaur \\ MPT Student, DAV Institute of \\ Physiotherapy and Rehabilitation, \\ Jalandhar, Punjab
}

\author{
Dr. Jaspinder Kaur \\ Associate Professor, DAV Institute \\ of Physiotherapy and \\ Rehabilitation, Jalandhar, Punjab
}

\begin{abstract}
Background and Objective: According to world Health Organization (WHO) stroke is defined as "rapidly developing clinical sign of focal (or global) disturbance of cerebral function lasting more than 24 hours or leading to death, with no apparent cause other than vascular origin. ${ }^{1}$ Focal neurological deficits must persists for at least 24 hours, motor deficits are characterized by paralysis (hemiplegia) or weakness (hemiparesis), typically on the side of the body opposite site of lesion.
\end{abstract}

Materials and Methods: The study was performed among 30 patients of both genders, aged 45-60 years. Subjects were selected on the basis of inclusion criteria and randomly divided into two groups by convenience sampling and allocating alternate patient group A and group B, 15 in each group. Group A was treated with Repetitive Facilitation Exercises (RFE), Group B was treated with Hand Arm Bimanual Intensive Training (HABIT). Baseline assessments were taken using WMFT and FMA and data was analyzed.

Results: The groups showed significant differences in WMFT and FMA variables. But on comparing the mean of both the groups: there was no significant difference between both the groups.
Conclusion: This study concluded that RFE and HABIT both are effective in treatment of patients with hemiparesis.

Keywords: Hemiparesis, RFE, HABIT, WMFT, FMA

\section{INTRODUCTION}

According to world Health Organization (WHO) stroke is defined as "rapidly developing clinical sign of focal (or global) disturbance of cerebral function lasting more than 24 hours or leading to death, with no apparent cause other than vascular origin. ${ }^{1} \mathrm{Focal}$ neurological deficits must persists for at least 24 hours, motor deficits are characterized by paralysis (hemiplegia) or weakness (hemiparesis), typically on the side of the body opposite site of lesion. Strokes can be classified into two major categories: ischemic or hemorrhagic. ${ }^{2}$ Stroke is the most common cause of disability of survivors, an estimated one third will be functionally dependent after one year experiencing with difficulty of activities of daily living, ambulation and speech, with $30 \%$ to $66 \%$ of individuals lose functional ability in more affected arm and hand. ${ }^{3}$ Functional recovery of the paretic upper extremity post stroke continue to be the one of the greatest challenges faced by the rehabilitation professionals, although most of the patients gain walking ability but fail to gain functional use of their arms and hands. ${ }^{4}$ Upper extremity rehabilitation protocol for stroke hemiparesis have traditionally primary on the paretic 
limb with unilateral strengthening exercise, neuromuscular re-education and functional training. Upper extremity paresis is leading cause of function disability after stroke (self-care, feeding $\&$ dressing). ${ }^{5}$ A number of instruments have been developed to evaluate functional recovery after stroke. Some examples of instruments for determining upperextremity functional ability are the ChedokeeMcMaster (CM) assessment, the Fugl-Meyer (FMA) assessment and the Wolf Motor Function Test (WMFT) assessment. ${ }^{6}$

It has been seen that the recovery in lower limbs is better than upper limb in post stroke patients. It is because both lower extremities are equally required for normal gait, by adopting compensatory gait strategies; patients can perform his activities to some extent. Patient prefers to use unaffected hand to complete the tasks and neglect the use of affected upper limb. Previous studies have shown the effectiveness of Repetitive facilitation exercises (RFEs) and Hand arm bimanual intensive training (HABIT) in improvement of upper extremity in post stroke hemiparetic patients. This study explored the effectiveness of Repetitive facilitation exercises (RFEs) and Hand arm bimanual intensive training (HABIT).

Wolf motor function test was designed to assess the motor ability of patients with moderate to severe upper extremity motor deficits in the laboratory and clinic $^{7}$

\section{The upper-extremity section of Fugl-Meyer (FMA)} scale was designed to assess the motor ability of patients. It includes 27 grades motor function on the ICF level of body functions (muscle and movement functions). A 3-point ordinal scale is used to score 33 items, with a maximum score of 66 . Good reliability and validity have been reported. ${ }^{8}$

Kazumi Kawahira et al (2010) conducted a study to evaluate the effects on the hemiplegic upper limb of repetitive facilitation exercise (RFEs) using a novel facilitation technique, in which the patient's intention to move the hemiplegic upper limb or finger was followed by realization of the movement using multiple sensory stimulations. 23 stroke patients were enrolled in which 2- week RFE sessions (100 repetitions each of 5-8 types of facilitation exercise per day) were altered with 2 -week conventional rehabilitation (CR) sessions, for a total of four sessions, for a total of four sessions. The simple test for Evaluating hand function (STEF) score, which evaluates the ability of manipulating objects, in both groups improved during both sessions. After the second week RFE and CR sessions, both groups showed little further improvement except in the STEF score. ${ }^{9}$

Jill Whitall et al (2000) investigated the result of bilateral arm training with rhythmic auditory cueing will improve motor function in the hemiparetic arm of stroke patients. They determined the effects of 6 weeks of BATRAC on 14 patients with chronic hemiparetic stroke immediately after training and at 2 months after training. Four 5- minute periods per session (3 times per week) of BATRAC were performed with the use of a custom designed arm training machine. At last, they concluded that with six weeks of bilateral arm training with rhythmic auditory cueing (BATRAC) improves functional motor performance of the paretic upper extremity as well as a few changes in isometric strength and range of motion. These benefits are largely sustained at weeks after training cessation. ${ }^{10}$

\section{Methodology}

The study design was quasi experimental and comparative in nature. 30 hemiparetic subjects aged between 45-60 years were included on the basis of inclusion criteria: Sub acute stroke patients (30 days 6 months), both male and female subjects, patients with hemorrhagic and ischemic stroke, patients having minimum 10 degree wrist extension and finger extension actively for habit group, patients with mini mental state examination score of 24 or more, patients demonstrate independent full range of motor of upper limb passively, patient is able to maintain sitting balance. Patients were excluded from the study on the basis of exclusion criteria: visual-perceptual problems, participating in any experimental study which include upper limb treatment, communication barrier, patients with behavioural disturbances and psychologically non cooperative, shoulder pain insensitive to standard therapy, hand swelling sufficient to prevent fist formation, painful arthritis of the shoulder or wrist and finger joint, excessive spasticity, defined as having a score of 3 on the Modified Ashworth Spasticity Scale, participating in any experimental rehabilitation or drug studies, severe 
sensory disturbance of affected upper limb, dementia, patients with history of upper limb surgery.

Sampling method was convenient sampling. A written consent was taken from the patients. Patients were divided into 2 groups:- Group A:Repetitive Facilitation Exercises (RFE) and Group B: Hand Arm Bimanual Intensive Training (HABIT). In the study, assessment of patient was done by Wolf motor function test (WMFT) and Fugl - Mayer assessment for upper limb (FMA-UE).

Subjects received the treatment for 5 session/week for consecutive 2 weeks (total 10 sessions). Post reading was taken at 2 weeks (after 10 sessions).

\section{Group A:}

The RFE session consisted of stretch reflex by rapid passive stretching of the muscles and the skin-muscle reflex which is induced by tapping or rubbing on the muscles in a specific posture that maintained the tension in the targeted muscles. Each repetitive exercise was given for 100 repetitions per session for 2 weeks.

\section{Group B:}

The activities were divided into five movement categories that focus on the distal extremity. Total time for each set of movements was 10 minutes and rest for 2 minutes. The total treatment time was 1hours.

\section{Statistical Analysis}

Unpaired t-test was used to compare the scores of baseline assessments between the two groups to find out whether the two groups were comparable or not. The groups have $p>0.05$ are comparable. Paired t-test was used to compare the variables within the groups. Unpaired t-test was used to compare the variables between the groups.

\section{INTERPRETATION}

Table1: Mean and SD of age of the subjects for the Group A and Group B

\begin{tabular}{|c|c|c|c|}
\hline Age & Mean + SD & t- Value & Level of Significance \\
\cline { 1 - 2 } Group A & $51.27 \pm 4.95$ & & 0.837 \\
Group B & $51.67 \pm 5.64$ & 0.206 & NS \\
\hline
\end{tabular}

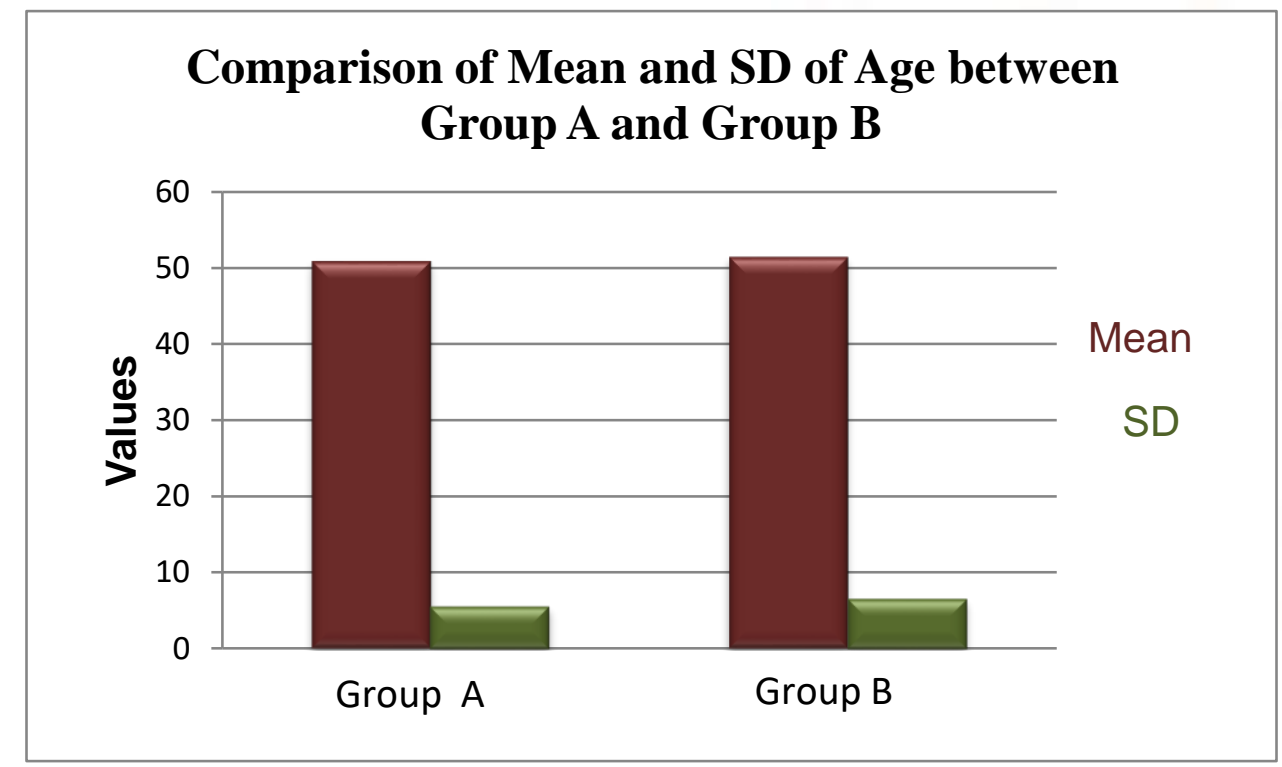


Table2: WMFT within group A

\begin{tabular}{|l|l|l|l|l|}
\hline \multicolumn{2}{|c|}{ WMIFT } & Mean \pm SD & t-value & $\begin{array}{l}\text { Level of } \\
\text { Sig. }\end{array}$ \\
\hline \multirow{2}{*}{ Group A } & Pre Value & $162 \pm 6.15$ & \multirow{2}{*}{15.80} & $\begin{array}{l}0.0001 \\
\text { S }\end{array}$ \\
\cline { 2 - 3 } & Post Value & $140.9 \pm 5.26$ & & \\
\hline
\end{tabular}

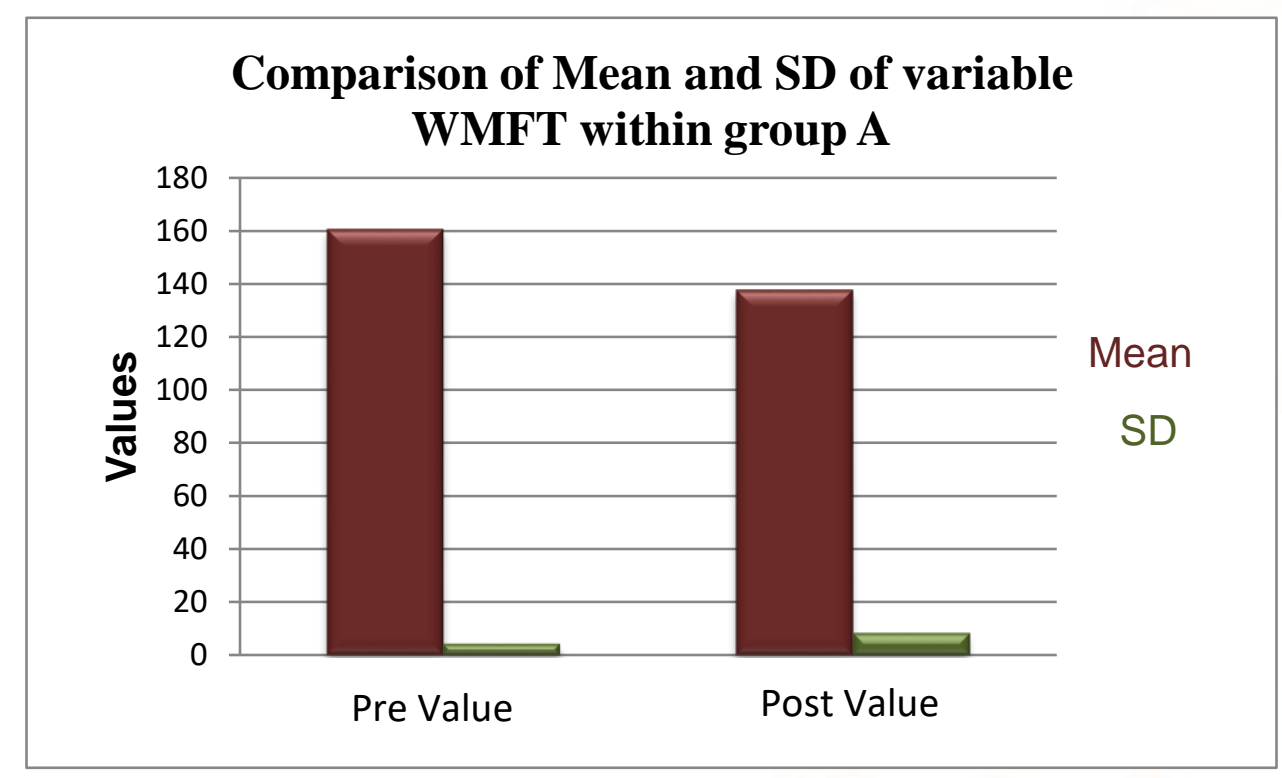

Table 3: WMFT within group B

\begin{tabular}{|l|l|l|l|l|}
\hline \multicolumn{2}{|c|}{ WMIFT } & Mean \pm SD & t-value & $\begin{array}{l}\text { Level of } \\
\text { Sig. }\end{array}$ \\
\hline \multirow{2}{*}{ Group B } & Pre Value & $160.6 \pm 4.15$ & \multirow{2}{*}{11.84} & $\begin{array}{l}0.0001 \\
\text { S }\end{array}$ \\
\cline { 2 - 4 } & Post Value & $137.8 \pm 8.08$ & & \\
\hline
\end{tabular}

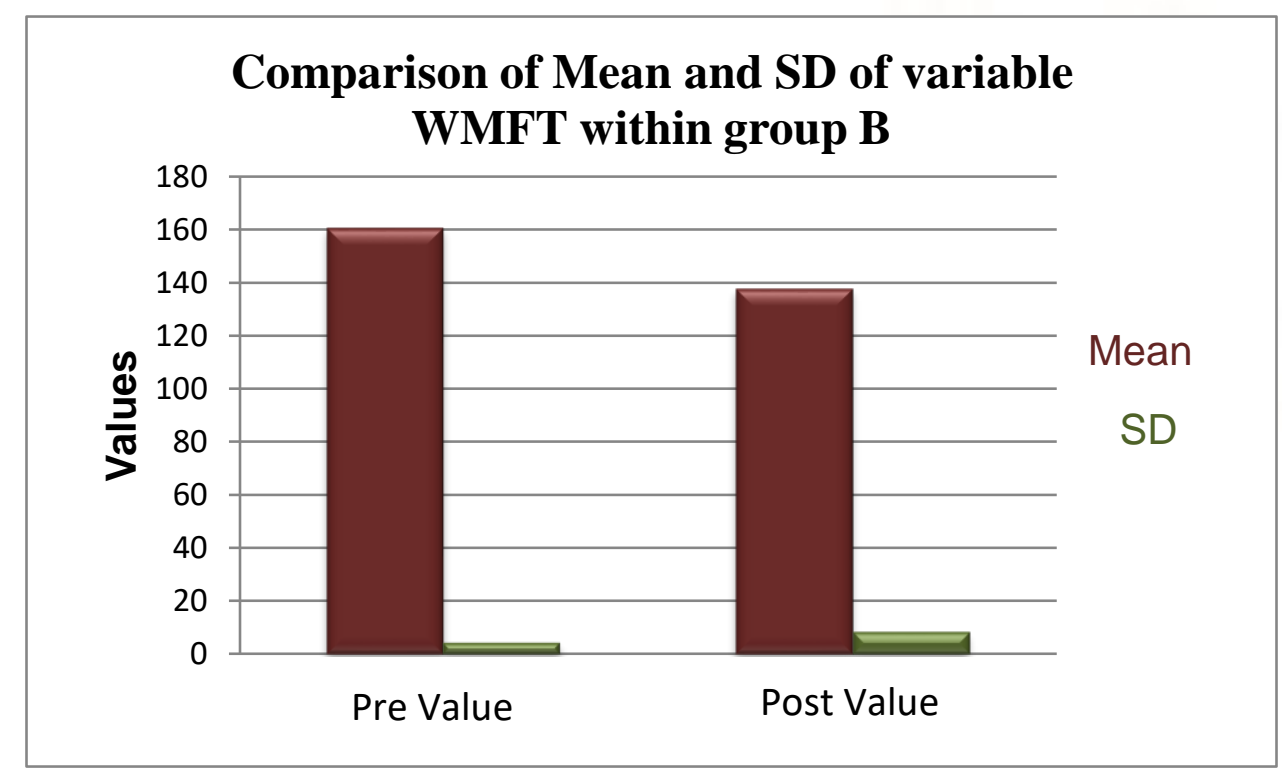


Table 4: FMA - UE within group A

\begin{tabular}{|l|l|l|l|l|}
\hline \multicolumn{2}{|c|}{ FMA - UE } & \multicolumn{1}{|c|}{ Mean \pm SD } & \multicolumn{1}{|c|}{ t-value } & \multicolumn{1}{|c|}{$\begin{array}{c}\text { Level of } \\
\text { Sig. }\end{array}$} \\
\hline \multirow{2}{*}{ Group A } & Pre Value & $35.73 \pm 5.48$ & \multirow{2}{*}{11.14} & 0.0001 \\
& & & S \\
\cline { 2 - 5 } & Post Value & $47.33 \pm 5.07$ & & \\
\hline
\end{tabular}



Table5: FMA - UE within group B

\begin{tabular}{|l|l|l|l|l|}
\hline \multicolumn{2}{|c|}{ FMA - UE } & \multicolumn{1}{|c|}{ Mean \pm SD } & \multicolumn{1}{|c|}{ t-value } & \multicolumn{1}{|c|}{$\begin{array}{c}\text { Level of } \\
\text { Sig. }\end{array}$} \\
\hline Group B & Pre Value & $36.80 \pm 5.62$ & 11.01 & $\begin{array}{l}0.0001 \\
\text { S }\end{array}$ \\
\hline
\end{tabular}




Table 6: WMFT between group A and group B

\begin{tabular}{|c|c|c|c|c|}
\hline \multicolumn{2}{|c|}{ WMFT } & Mean \pm SD & t-value & $\begin{array}{c}\text { Level of } \\
\text { Sig. }\end{array}$ \\
\hline \multirow{2}{*}{$\begin{array}{c}\text { Pre Test } \\
(\text { O Day) }\end{array}$} & Group A & $162 \pm 6.15$ & & 0.492 \\
\cline { 2 - 3 } & Group B & $160.6 . \pm 4.15$ & 0.695 & NS \\
\hline $\begin{array}{c}\text { Post Test } \\
(2 \text { Weeks) }\end{array}$ & Group A & $140.9 \pm 5.26$ & & 0.228 \\
\cline { 2 - 3 } & Group B & $137.8 \pm 8.08$ & 1.232 & NS \\
\hline
\end{tabular}



Table 7: FMA - UE between group A and group B

\begin{tabular}{|l|l|l|l|l|}
\hline \multicolumn{2}{|c|}{ FMA - UE } & \multicolumn{1}{|c|}{ Mean \pm SD } & \multicolumn{1}{|c|}{$\begin{array}{l}\text { Level of } \\
\text { Sig. }\end{array}$} \\
\hline $\begin{array}{l}\text { Pre Test } \\
\text { ( D Day) }\end{array}$ & Group A & $35.73 \pm 5.48$ & 0.526 & $\begin{array}{l}0.603 \\
\text { NS }\end{array}$ \\
\cline { 2 - 5 } & Group B & $36.80 . \pm 5.62$ & & $\begin{array}{l}0.607 \\
\text { NS }\end{array}$ \\
\hline $\begin{array}{l}\text { Post Test } \\
(2 \text { Weeks) }\end{array}$ & Group A & $47.33 \pm 5.07$ & 0.519 & \\
\hline
\end{tabular}




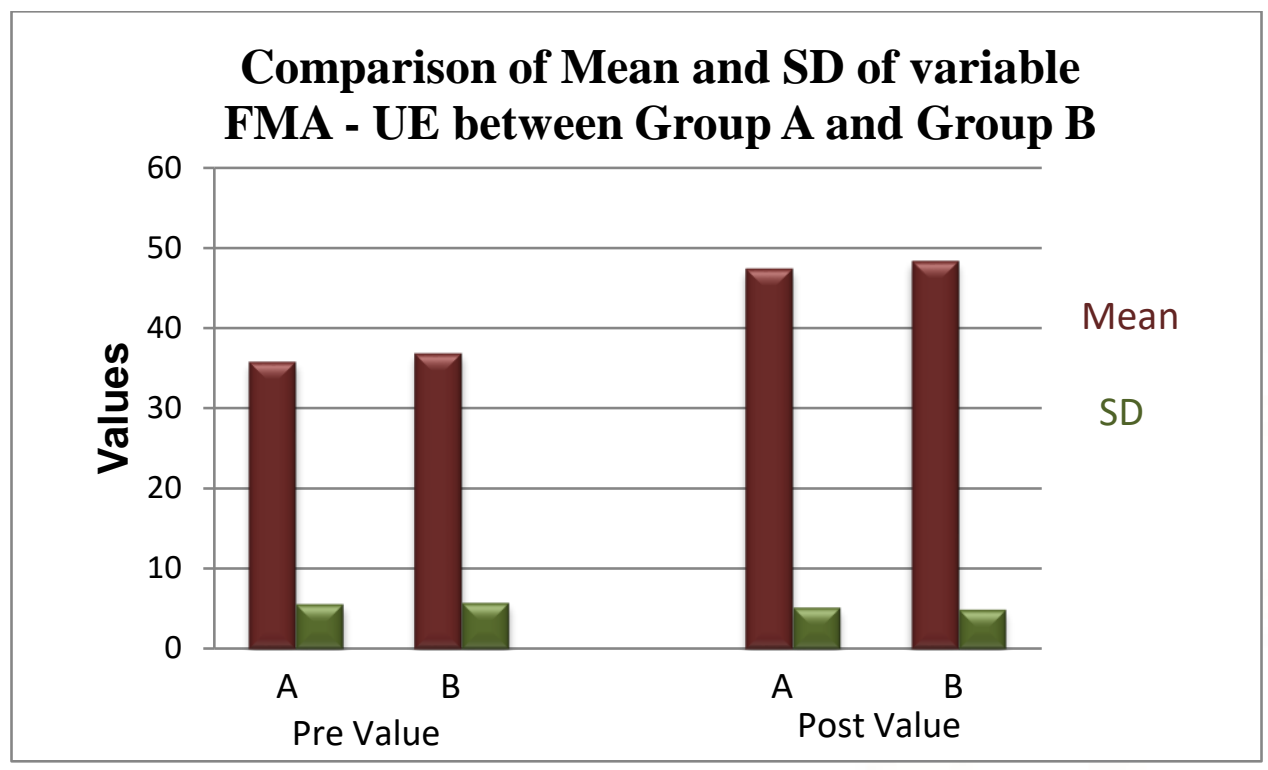

Table 1 showed that there was no significant difference in the age group. So, the groups were comparable. Table 2 showed that there were significant changes of WMFT variable within the group A. Table 3 showed that there were significant changes of WMFT variable within the group B. Table 4 showed that there were significant changes of FMA variable within the group A. Table 5 showed that there were significant changes of FMA variable within the group B. Table 6 showed that there were not significant changes of WMFT variable between the groups. Table 7 showed that there were not significant changes of FMA variable between the groups.

\section{DISCUSSION}

The study was done to evaluate the effect of repetitive facilitation exercises and hand arm bimanual intensive training on wolf motor function test and fugl-meyer for upper extremity in post stroke hemiparetic patients. The hypothesis was that the significant change in the effectiveness between Repetitive facilitation exercises (RFEs) and Hand arm bimanual intensive training (HABIT) on upper limb functions in post stroke hemiparetic patients.

It is necessary to know that the functional recovery of the paretic Upper extremity continues to be the one of greatest challenge. Patient with history of stroke can gain walking ability but fail to use of their upper extremity function ${ }^{11}$ The massed practice design used to improve the function of individual with hemiparesis by constraining the less affected arm ${ }^{.12}$
The effects of the RFEs used in the novel facilitation technique that was developed on the isolation from synergy and the manipulation of objects of the hemiplegic upper limb. The novel facilitation methods used in previous studies were designed to give focused sufficient physical stimulation to realize the patient's intended movements. These physical stimulation were the stretch reflex, skin-muscle reflex, and alpha and gamma linkage, which were induced by tapping or rubbing the muscles, rapid passive stretching of the muscle or slight resistance against the intended movements. In the present study we were followed the same repetitive facilitation exercises regimen with 5 movements rather than 8 movements ${ }^{13}$

In any case, motor functional recovery of a hemiplegic finger requires more repetitive voluntary upper limb and finger movements that are isolated from synergy by facilitation techniques, because it is difficult for patients to move the hemiplegic upper limb alone, especially the fingers. RFEs for the hemiplegic upper limb improved the functional recovery when voluntary movements were elicited by the novel facilitation technique, although patients who did not show the effects of this facilitation might gain little from RFEs. Recent studies have shown that brain plasticity contributes to functional recovery from paralysis. Forced use of the hemiplegic hand after brain damage in adult primates and finger tracking training in adult humans who suffered a stroke led to changes in motor representation in the primary motor cortex ${ }^{.14}$ 
Regarding the activities in bimanual training, Larisa R. Hoffman et al(2007) gave bimanual training to cervical spinal cord injury patient who had complete motor spinal cord injury at the level of $\mathrm{C}_{6}$. The training include five activities -Grasp, Grasp with rotation, finger isolation, pinch and pinch with rotation. There is significant improvement seen in both the upper limbs in the present study, same activities were taken to see the improvement in hand function. ${ }^{15}$

One of the previous study on hand arm bimanual intensive training was conducted by Jeanne Charles, Andrew M Gordon et al(2006) ${ }^{16}$ found that it is becoming increasingly clear that the key to eliciting improvement in function is to provide sufficient practice. However, rather than just focusing on repetition, the practice must be structured, based on how the central nervous system responds during learning; i.e. optimal responses with increasing movement complexity and provision of motivation and reward. ${ }^{17}$ Thus, appropriate intensity must be provided with these guidelines in mind. There is rapid development of ipsilateral and contralateral corticospinal projections from the undamaged hemispheres. The corticospinal system underlying skilled manipulative control is capable of reorganization after damage, probably underlying recovery of function. ${ }^{18}$ Ipsilateral pathways have been shown to be implicated in the recovery of function after stroke. Thus, tasks recruiting the ipsilateral pathways such as symmetrical bilateral movements may be beneficial. ${ }^{19}$

\section{Wolf motor Function Test (WMFT)}

There were significant changes in the effectiveness of different treatments given by repetitive facilitation exercises and hand arm bimanual training on wolf motor function test (WFMT) on post stroke hemiparetic patients. All subjects treated with 60 minutes RFE showed decreased WMFT score (140.9 \pm 5.26) after 2 weeks, which were statistically significant from baseline. On the other hand, all subjects who received 60 minutes hand arm bimanual intensive training (HABIT) protocol also showed decreased WMFT score $(137.8 \pm 8.08)$ after 2 weeks treatment, which were statistically significant from baseline. When compared both the groups, both the treatments were equally effective in context of wolf motor function test (WMFT). The result of this study was supported by Kawahira K. and Shimodozono $M$ et al (2010) ${ }^{9}$ who demonstrated the use of RFEs that might have played a role in promoting motor functional improvement is the elicitation of voluntary movements of the hemiplegic limb.

The results of this study were in concordance with the results of Kumar A,Singh P. et al (2011) ${ }^{20}$ who demonstrated significant improvement in WMFT within the group comparison. The study found that when one limb must move further or aimed towards a smaller target compared to the other, the behaviour of one hand is affected by the task requirement of contralateral hand. This is known as assimilation effect. Assimilation effect are usually asymmetric in nature such that the limb performing more difficult task impacts the limb performing a easier task to a greater extent than the converse.

\section{Fugl Meyer Assessment for Upper Extremity (FMA - UE)}

There were significant changes in the effectiveness of both the techniques on Fugl Meyer Assessment for Upper Extremity (FMA - UE) on post stroke hemiparetic patients. All subjects treated with 60 minutes RFE showed decreased FMA - UE score $(47.33 \pm 5.07)$ after 2 weeks, which were statistically significant from baseline. On the other hand, all subjects who received 60 minutes hand arm bimanual intensive training (HABIT) protocol also showed decreased FMA - UE score $(48.27 \pm 4.77)$ after 2 weeks treatment, which were statistically significant from baseline. When compared both the groups, both the treatments were equally effective in context of Fugl Meyer Assessment for Upper Extremity (FMA UE). The result of this study was also supported by John Chae et al. (1998) $)^{21}$ whoanalysedthe score of fugl-meyer revealed significantly greater motor improvement for the neuromuscular stimulation group at 4 weeks after treatment and at 12 weeks after treatment.The result of this study was also supported by Jill Whital et al (2000 $)^{22}$ whoconducted a study on bilateral movements and concluded that practicing bilateral movement in synchrony may result in facilitation effect from non paretic arm to paretic arm, when bimanual movements are initiated simultaneously. Both arms act as unit that supercedes the individual arm action, indicating that both arms are linked as a co-ordinate unit in the brain ${ }^{.23}$

\section{CONCLUSION}

Based on the data analysis it can be interpreted that Repetitive facilitation Exercises and Hand Arm Bimanual Intensive Training are equally effective on 
outcome parameters in patients with hemiparesis. Therapist can choose any of the treatment technique for the betterment of the hemiparesis patients in context of upper limb recovery.

\section{Limitations}

$>$ Small sample size

$>$ Absence of randomization of sampling and appropriate control group

$>$ Follow Up

\section{Recommendations}

$>$ Random sampling should be there.

$>$ This study can be done on larger sample size.

$>$ This study can also be done on younger age group.

$>$ Gender specific study can also be done.

$>$ This study can be done in different phases of stroke (Acute and chronic)

\section{Ethical clearance}

No ethical clearance required.

\section{Source of funding}

No funding was required to carry out this work.

\section{Acknowledgement}

We are thankful to Dr Jitendra Shrama, Principle, DAV Institute of Physiotherapy and Rehabilitation, Jalandhar, Punjab-144008.

\section{Conflicts of Interest}

Authors declare that there is no conflict of interest.

\section{Reference}

1) Patrica A. Downie, Dane Cicely Saunder, 'Cash Text book of neurology for physiotherapy $4{ }^{\text {th }} \mathrm{Ed}$,' Japee brothers , pp.240.

2) Susan B.O Sullivan 2007, 'Physical rehabilitation $5^{\text {th }}$ Edition,' vol 382, pp.705-706.

3) Sharp SA, Brouwer BJ. Isokinetic strength training of the hemiparetic knee: effects on function and spasticity. Archives of physical medicine and rehabilitation. 1997 Nov 1;78(11):1231-6.

4) Merletti R, Andina A, Galante M, Furlan I. Clinical experience of electronic peroneal stimulators in 50 hemiparetic patients. Scandinavian journal of rehabilitation medicine. 1979;11(3):111-21.

5) Basmajian JV, Gowland CA, Finlayson MA, Hall AL, Swanson LR, Stratford PW, Trotter JE,
Brandstater ME. Stroke treatment: comparison of integrated behavioral-physical therapy vs traditional physical therapy programs. Archives of Physical Medicine and Rehabilitation. 1987 May;68(5 Pt 1):267-72.

6) Wolf SL, Catlin PA, Ellis M, Archer AL, Morgan B, Piacentino A. Assessing Wolf motor function test as outcome measure for research in patients after stroke. Stroke. 2001 Jul 1;32(7):1635-9.

7) Wolf SL, Winstein CJ, Miller JP, Taub E, Uswatte G, Morris D, Giuliani C, Light KE, NicholsLarsen D, Excite Investigators. Effect of constraint-induced movement therapy on upper extremity function 3 to 9 months after stroke: the EXCITE randomized clinical trial. Jama. 2006 Nov 1;296(17):2095-104.

8) Fugl-Meyer AR, Jääskö L, Leyman I, Olsson S, Steglind S. The post-stroke hemiplegic patient. 1. a method for evaluation of physical performance. Scandinavian journal of rehabilitation medicine. 1975;7(1):13-31.

9) Kawahira K, Shimodozono M, Etoh S, Kamada K, Noma T, Tanaka N. Effects of intensive repetition of a new facilitation technique on motor functional recovery of the hemiplegic upper limb and hand. Brain Injury. 2010 Sep 1;24(10):120213.

10) Whitall J, Waller SM, Silver KH, Macko RF. Repetitive bilateral arm training with rhythmic auditory cueing improves motor function in chronic hemiparetic stroke. Stroke. 2000 Oct 1;31(10):2390-5.

11) Bütefisch $C$, Hummelsheim $H$, Denzler P, Mauritz $\mathrm{KH}$. Repetitive training of isolated movements improves the outcome of motor rehabilitation of the centrally paretic hand. Journal of the neurological sciences. 1995 May 31;130(1):59-68.

12) Morris DM, Crago JE, DeLuca SC, Pidikiti RD, Taub E. Constraint-induced movement therapy for motor recovery after stroke. Neurorehabilitation. 1997 Jan 1;9(1):29-43.

13) Woldag H, Waldmann G, Heuschkel G, Hummelsheim $\mathrm{H}$. Is the repetitive training of complex hand and arm movements benefi cial for motor recovery in stroke patients?. Clinical Rehabilitation. 2003 Nov; 17(7):723-30.

14) Blanton S, Wolf SL. An application of upperextremity constraint-induced movement therapy in a patient with subacute stroke. Physical therapy. 1999 Sep 1;79(9):847-53. 
15) Hoffman LR, Field-Fote EC. Cortical reorganization following bimanual training and somatosensory stimulation in cervical spinal cord injury: a case report. Physical therapy. 2007 Feb 1;87(2):208-23.

16) Charles J, Gordon AM. Development of hand-arm bimanual intensive training (HABIT) for improving bimanual coordination in children with hemiplegic cerebral palsy. Developmental medicine and child neurology. 2006 Nov;48(11):931-6.

17) Kleim JA, Hogg TM, VandenBerg PM, Cooper NR, Bruneau R, Remple M. Cortical synaptogenesis and motor map reorganization occur during late, but not early, phase of motor skill learning. Journal of Neuroscience. 2004 Jan 21;24(3):628-33.

18) Eyre JA. Development and plasticity of the corticospinal system in man. Neural plasticity. 2003;10(1-2):93-106.

19) Stinear JW, Byblow WD. Rhythmic bilateral movement training modulates corticomotor excitability and enhances upper limb motricity poststroke: a pilot study. Journal of Clinical Neurophysiology. 2004 Mar 1;21(2):124-31.

20) Gorgos KS, Wasylyk NT, Van Lunen BL, Hoch MC. Inter-clinician and intra-clinician reliability of force application during joint mobilization: a systematic review. Manual therapy. 2014 Apr 30;19(2):90-6.

21) Chae J, Bethoux F, Bohinc T, Dobos L, Davis T, Friedl A. Neuromuscular stimulation for upper extremity motor and functional recovery in acute hemiplegia. Stroke. 1998 May 1;29(5):975-9.

22) Whitall J, Waller SM, Silver KH, Macko RF. Repetitive bilateral arm training with rhythmic auditory cueing improves motor function in chronic hemiparetic stroke. Stroke. 2000 Oct 1;31(10):2390-5.

23) Fowler B, Duck T, Mosher M, Mathieson B. The coordination of bimanual aiming movements: evidence for progressive desynchronization. The Quarterly journal of experimental psychology. $1991 \quad$ May 1; 43(2):205-21. 